\title{
Usefulness of the metabolic syndrome criteria as predictors of insulin resistance among obese Korean women
}

\author{
Kayoung Lee*
}

Department of Family Medicine, Inje University Busan Paik Hospital, 633-165 Kaegum-dong, Pusan Jin-Gu, Busan 614-735, Republic of Korea

Submitted 10 March 2009: Accepted 20 July 2009: First published online 26 August 2009

\begin{abstract}
Objective: To investigate the ability of each metabolic syndrome (MetS) criterion, defined by the International Diabetes Federation, to predict insulin resistance (IR). Design: A cross-sectional study. IR was defined as homeostasis model assessment of IR (HOMA-IR) $\geq 3 \cdot 04$. The MetS criteria considered were TAG $\geq 1 \cdot 69 \mathrm{mmol} / \mathrm{l}$, HDL cholesterol (HDL-C) $<1.29 \mathrm{mmol} / 1$, blood pressure $(\mathrm{BP}) \geq 130 / 85 \mathrm{mmHg}$ and fasting glucose $(\mathrm{FG}) \geq 5.6 \mathrm{mmol} / 1$.

Setting: Busan, South Korea.

Subjects: Ninety-six apparently healthy Korean women (mean age 42 (SD 10.6) years) with abdominal obesity (waist circumference (WC) $\geq 80 \mathrm{~cm}$ ) were studied. Results: Of the ninety-six obese women, $11 \%$ were insulin-resistant and 33\% fulfilled the criteria for IDF-defined MetS. Glucose and TAG were more likely to predict IR than BP and HDL-C when assessed using receiver-operating characteristic curves, multiple regression and multiple logistic regression analyses. Of the variation in HOMA-IR, TAG, FG, WC and age explained $42 \%$. High FG was independently associated with the presence of IR (OR $=8 \cdot 6,95 \%$ CI 1.8, 41.8) even after adjusting for other components of MetS. The positive predictive value and positive likelihood ratio to detect IR were the highest for the FG criterion (33\% and $3 \cdot 9$, respectively), followed by TAG $(28 \%, 3 \cdot 0)$, BP $(19 \%, 1 \cdot 8)$ and HDL-C criteria $(18 \%, 1 \cdot 7)$. The IDF definition of MetS exhibited a positive predictive value of $29 \%$ and a positive likelihood ratio of $3 \cdot 1$.

Conclusions: Of the MetS criteria, high FG and high TAG seem to be more suitable for identifying obese women with IR than high BP and low HDL-C.
\end{abstract}

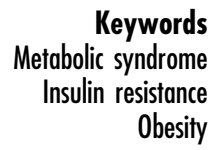

Insulin resistance (IR) is a physiological abnormality that increases the risk of developing type 2 diabetes mellitus and CVD, and plays a central role in the development of the metabolic syndrome (MetS), which is a cluster of lipid and non-lipid CVD risk factors of metabolic origin ${ }^{(1)}$. According to evolving views of the pathogenesis of the syndrome, MetS criteria have changed. While the WHO task force on diabetes requires IR as one criterion of MetS, the National Cholesterol Education Program Adult Treatment Panel III and the International Diabetes Federation (IDF) selected five criteria: high fasting glucose (FG), high fasting TAG, low HDL cholesterol (HDL-C), high blood pressure (BP) and high waist circumference (WC) ${ }^{(2,3)}$. Determining whether the MetS criteria have better prediction of IR would be useful because direct measures of IR are not clinically practical.

Obese women are at risk for $\mathrm{IR}^{(4)}$; however, a subset of obese women are not insulin-resistant and are metabolically healthy ${ }^{(5)}$. Additionally, a previous study suggests that nutritional therapy for obese women may be detrimental in improving insulin sensitivity depending on their underlying metabolic health status ${ }^{(6)}$. Therefore, assessing IR using more easily applicable MetS criteria would be useful to identify obese women who need weight loss to improve insulin sensitivity. There is evidence that MetS criteria predict IR with moderate sensitivity and high specificity among obese individuals ${ }^{(7)}$, but it is not clear whether the ability of each MetS criterion in the prediction is the same ${ }^{(1,8)}$.

Therefore, the objective of the present study was to investigate the ability of the IDF-defined MetS criteria to predict IR among obese Korean women.

\section{Experimental methods}

\section{Study participants}

Study participants were ninety-six apparently healthy Korean women (mean age 42 (sD 10.6) years) with central obesity (WC $\geq 80 \mathrm{~cm})^{(3)}$ who were non-randomly recruited using posters and flyers in two hospitals in Busan, South Korea. Exclusion criteria for participation in the study were those who had unstable weight over the past 
12 weeks, a significant chronic disease or were taking medications (anti-obesity medications, steroids, thyroid hormone, antipsychotics, antidepressants, anxiolytics, laxatives, oral contraceptives, beta-blockers, diuretics, oral hypoglycaemic agents, insulin, or hormone replacement therapy) that could affect body weight and body water content. Twenty-six women were in menopause status and three women had undergone hysterectomy. The Institutional Review Boards of Busan Paik Hospital, Busan, South Korea, approved all methods performed in the study.

\section{Measurements of metabolic variables, adiposity and insulin resistance}

Blood pressure and concentrations of total cholesterol (TC), LDL cholesterol (LDL-C), HDL-C, TAG, TAG:HDL-C, FG, insulin and high-sensitivity C-reactive protein (hsCRP) were assessed as metabolic variables. The ratio of TAG to HDL-C has been used to reflect IR in some studies $^{(7)}$. Adiposity measurements included were WC, BMI, percentages of total fat mass and android fat mass, and areas of subcutaneous abdominal fat (SAF) and intraabdominal fat (IAF).

BP was measured twice using a standard manual sphygmomanometer with the participants in the sitting position and the average values of BP were used. The subjects' fasting status ( $\geq 12 \mathrm{~h}$ ) was confirmed by research assistants before blood samples were obtained. Plasma glucose was assayed using the hexokinase enzymatic method, and insulin concentration was determined by RIA. Serum TC, HDL-C, LDL-C and TAG were measured by homogeneous assay and enzymatic methods. Latex immune complex turbidimetry was used to measure hs-CRP. The following criteria were used to define MetS: TAG $\geq 1.69 \mathrm{mmol} / 1, \quad$ HDL-C $<1.29 \mathrm{mmol} / 1, \quad \mathrm{BP} \geq 130 /$ $85 \mathrm{mmHg}$ and $\mathrm{FG} \geq 5.6 \mathrm{mmol} / \mathrm{l}^{(3)}$.

Homeostasis model assessment of IR (HOMA-IR index) was chosen as a surrogate marker of IR. HOMA-IR was calculated as fasting insulin $(\mu \mathrm{U} / \mathrm{ml})$ multiplied by $\mathrm{FG}$ $(\mathrm{mmol} / \mathrm{l})$ divided by $22 \cdot 5^{(9)}$. The cut-off value of HOMAIR for classifying IR was $3 \cdot 04^{(10)}$.

Research assistants measured participants' WC at a level midway between the lowest lateral border of the ribs and the uppermost lateral iliac crest with the participants standing. Circumference was measured twice to the nearest $0 \cdot 1 \mathrm{~cm}$ and the mean value of the two measurements calculated. The reproducibility of the two measurements revealed a high intra-class correlation coefficient $(0 \cdot 98)$.

Measurements were also taken for participants' body weight (to the nearest $0 \cdot 1 \mathrm{~kg}$ ) and height (to the nearest $0 \cdot 1 \mathrm{~cm}$ ) using a digital balance (Tanita Co., Seoul, South Korea) and stadiometer (Samwha Co., Seoul, South Korea), as they wore light clothing but no shoes. BMI was calculated by dividing the weight by the square of height $\left(\mathrm{kg} / \mathrm{m}^{2}\right)$.

Dual-energy X-ray absorptiometry scans using Lunar Prodigy version 8.50 (Lunar Radiation Corporation,
Madison, WI, USA) were used to measure percentage of total fat mass and percentage of android fat mass. Fat mass percentage was calculated as fat mass divided by (fat mass + lean mass + bone mineral content). The android region of interest was defined inferiorly at the pelvis cut line, superiorly above the pelvis cut line by $20 \%$ of the distance between the pelvis and neck cut, and laterally at the arm cut lines. The cut lines for the regions were manipulated manually by a technician. Areas of SAF and IAF were measured using computerized tomography (CT) scans of an abdominal slice at the level of the intervertebral space between the fourth and fifth lumbar vertebrae. A Siemens Somatome Plus-4 CT scanner (Siemens, Erlangen, Germany) and the RAPIDIA 3D program (Infinitt Technology, Seoul, South Korea) were used for image analyses. The SAF and IAF regions were separated at the middle of the rectal abdominal muscles on the abdominal slices. An attenuation interval between -190 and -30 Hounsfield units was set to indicate fat, and Voxel Q (Picker International Inc., Cleveland, OH, USA) was used to calculate the areas of SAF and IAF.

\section{Statistical analyses}

Insulin, TAG:HDL-C, HOMA-IR and hs-CRP were logarithmically transformed (ln(insulin), $\ln (\mathrm{TAG}: \mathrm{HDL}-\mathrm{C})$, $\ln$ (HOMA-IR) and $\ln$ (hs-CRP), respectively) to be normally distributed. The independent $t$ test or $\chi^{2}$ test was used to compare continuous variables or categorical variables between women with and without IR. Pearson correlations between $\ln$ (HOMA-IR) and metabolic variables, including adiposity measurements, were calculated. The predictive ability of each MetS component and adiposity measures on IR were analysed using receiveroperating characteristic (ROC) curve analyses to calculate the area under the ROC curve (AUC) for identifying IR. Stepwise multiple regression analyses were conducted to construct models most highly explaining $\ln$ (HOMA-IR) from MetS components, including WC or other central obesity measures. Multiple logistic regression analyses were used to find independent relationships between IR and each MetS criterion after adjustment for other MetS criteria, including WC (as a continuous variable). Additional tests included to evaluate predictive ability of each MetS criterion on IR were kappa tests, sensitivity, specificity, likelihood ratio and predictive value. Differences were considered statistically significant when $P<0 \cdot 05$. All statistical analyses were performed using the SPSS statistical software package version $17 \cdot 0$ (release $17 \cdot 0 \cdot 0$; SPSS Inc., Chicago, IL, USA) and MedCalc version $9 \cdot 3 \cdot 0 \cdot 0$ (MedCalc Software, Mariakerke, Belgium).

\section{Results}

Of the ninety-six obese Korean women, $11 \%$ exhibited IR and $33 \%$ fulfilled the IDF definition of MetS. Table 1 
Table 1 Comparison of MetS components and adiposity measurements between apparently healthy obese women ( $n$ 96, mean age 42 years) with and without IR, Busan, South Korea

\begin{tabular}{|c|c|c|c|c|}
\hline & \multicolumn{2}{|c|}{ Women without IR ( $n$ 85) } & \multicolumn{2}{|c|}{ Women with IR ( $n$ 11) } \\
\hline & Mean & SD & Mean & SD \\
\hline WC (cm) & $89 \cdot 7$ & $7 \cdot 0$ & $93 \cdot 8^{\star}$ & $4 \cdot 2$ \\
\hline $\mathrm{FG}(\mathrm{mmol} / \mathrm{l})$ & $5 \cdot 11$ & 0.52 & $6 \cdot 11^{*}$ & $1 \cdot 17$ \\
\hline TAG (mmol/l) & $1 \cdot 12$ & 0.59 & $1 \cdot 78^{\star}$ & 1.06 \\
\hline $\mathrm{SBP}(\mathrm{mmHg})$ & $126 \cdot 0$ & $11 \cdot 7$ & $130 \cdot 5$ & $14 \cdot 8$ \\
\hline DBP $(\mathrm{mmHg})$ & $78 \cdot 0$ & $9 \cdot 4$ & $80 \cdot 7$ & $10 \cdot 0$ \\
\hline HDL-C (mmol/l) & $1 \cdot 47$ & 0.32 & $1 \cdot 37$ & 0.33 \\
\hline Ln(HOMA-IR) & 0.24 & 0.55 & $1 \cdot 56^{*}$ & $0 \cdot 37$ \\
\hline Ln(TAG:HDL-C) & -0.38 & 0.65 & $0 \cdot 12^{*}$ & $0 \cdot 76$ \\
\hline Ln(insulin) (pmol/l) & $3 \cdot 68$ & 0.53 & $4 \cdot 83^{*}$ & $0 \cdot 31$ \\
\hline $\mathrm{TC}(\mathrm{mmol} / \mathrm{l})$ & $4 \cdot 91$ & $0 \cdot 82$ & $5 \cdot 21^{*}$ & $0 \cdot 75$ \\
\hline LDL-C (mmol/l) & $2 \cdot 92$ & $0 \cdot 79$ & $3 \cdot 02$ & 0.90 \\
\hline Ln(hs-CRP) (mg/l) & $-0 \cdot 19$ & $1 \cdot 05$ & 0.46 & 0.96 \\
\hline Age (years) & 41.9 & $10 \cdot 1$ & $42 \cdot 3$ & $12 \cdot 0$ \\
\hline Menopause, $n(\%)$ & 22 & $26 \cdot 8$ & 4 & $36 \cdot 4$ \\
\hline BMI $\left(\mathrm{kg} / \mathrm{m}^{2}\right)$ & $27 \cdot 3$ & $3 \cdot 2$ & $28 \cdot 9^{*}$ & $3 \cdot 2$ \\
\hline Total body fat (\%) & $38 \cdot 6$ & 4.9 & $41 \cdot 3$ & $3 \cdot 9$ \\
\hline Android fat (\%) & $46 \cdot 3$ & $5 \cdot 4$ & $50 \cdot 0^{*}$ & $3 \cdot 4$ \\
\hline IAF area $\left(\mathrm{cm}^{2}\right)$ & $9 \cdot 6$ & $3 \cdot 7$ & $13 \cdot 6^{*}$ & $4 \cdot 1$ \\
\hline SAF area $\left(\mathrm{cm}^{2}\right)$ & $24 \cdot 8$ & $7 \cdot 2$ & $28 \cdot 9$ & $9 \cdot 9$ \\
\hline
\end{tabular}

MetS, metabolic syndrome; IR, insulin resistance; WC, waist circumference; FG, fasting glucose; SBP, systolic blood pressure; DBP, diastolic blood pressure, HDL-C, HDL cholesterol; HOMA-IR, homeostasis model assessment of IR; LDL-C, LDL cholesterol; TC, total cholesterol; hs-CRP, high-sensitivity C-reactive protein; IAF, intra-abdominal fat; SAF, subcutaneous abdominal fat. IR was defined as HOMA-IR $\geq 3.04^{(10)}$.

Mean values were significantly different from those of women without IR $\left(t\right.$ test): ${ }^{\star} P<0.05$.

presents comparisons of metabolic variables, including MetS components, and adiposity measurements between women with and without IR. Women with IR were more likely to have higher levels of FG, TAG, In(insulin), $\ln (\mathrm{TAG}: \mathrm{HDL}-\mathrm{C})$, WC, android fat and IAF area compared with women without IR, whereas levels of BP, HDL-C, ln(hs-CRP) and menopause status were not significantly different between the two groups.

Ln(HOMA-IR) was positively correlated with FG, TAG and $\ln$ (TAG:HDL) at similar levels, while it was not significantly correlated with BP. The correlations of In(HOMA-IR) with WC, BMI, android fat and IAF area also exhibited almost the same magnitude. Likewise, the AUC analyses showed that FG, TAG, $\ln$ (TAG:HDL), WC, android fat and IAF area predicted IR at similar levels, whereas BP and SAF area did not (Table 2).

Table 3 shows the best-fit models for $\ln$ (HOMA-IR). In model 1, among the five criteria, FG, TAG and WC were left as significant predicting variables, whereas BP and HDL-C were excluded from the model. Approximately $42 \%$ of the variation in $\ln$ (HOMA-IR) was able to be explained by these three variables plus age. Similarly, FG and TAG remained as significant predictors regardless of including different adiposity measures in the other models (IAF and SAF areas in model 2 or android fat in model 3).

The logistic regression model indicated an independent relationship between high FG and IR. Women with $\mathrm{FG} \geq 5.6 \mathrm{mmol} / 1$ were 8.6 times more likely to be insulinresistant compared with women having FG $<5.6 \mathrm{mmol} / \mathrm{l}$, after adjustment for other MetS criteria. High TAG was associated with the presence of IR before adjustment; however, the significance of the relationship disappeared after adjustment (Table 4).

Table 5 presents the ability of each MetS criterion and the IDF definition of MetS to identify obese women with IR. Kappa values exhibited lower associations between the variables $(\kappa=0 \cdot 4)$, although the kappa values for high FG and high TAG were significant. FG and TAG criteria demonstrated higher positive predictive value and positive likelihood ratio compared with HDL-C and BP criteria. Twenty-eight per cent and $33 \%$ of women meeting TAG and FG criteria, respectively, were insulin-resistant. In contrast, $92-94 \%$ of the women without any MetS criterion were not insulin-resistant. The odds of IR increased $3 \cdot 0$ and 3.9-fold if TAG and FG criteria, respectively were fulfilled, while the odds of IR decreased by $0 \cdot 6$ - and $0 \cdot 5$-fold if TAG and FG criteria, respectively, were not fulfilled. The IDF definition of MetS decreased positive predictive value and positive likelihood ratio but increased negative predictive value and negative likelihood ratio compared with those values for the FG criterion.

\section{Discussion}

The IDF definition for MetS is developed to provide tools to identify individuals who need lifestyle changes to decrease $\mathrm{IR}^{(11)}$. As obese women are more likely to be at higher risk for IR, MetS criteria with better precision in predicting IR would be clinically useful. Moreover, there is evidence that weight-loss management for obese women without IR results in aggravation of insulin sensitivity 
Table 2 Relationship of MetS components and adiposity measurements to $\ln (\mathrm{HOMA}-\mathrm{IR})$ in apparently healthy obese women ( $n 96$, mean age 42 years), Busan, South Korea

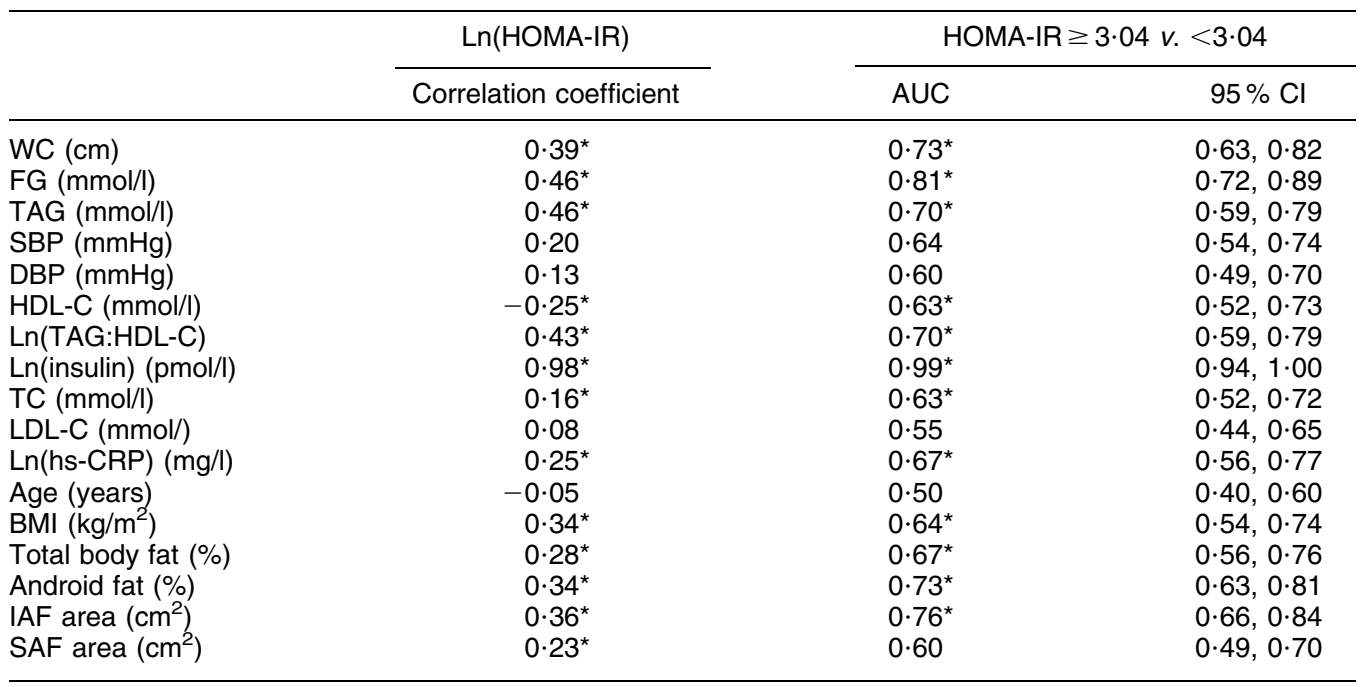

MetS, metabolic syndrome; HOMA-IR, homeostasis model assessment of insulin resistance; AUC, area under the receiveroperating characteristic curve; WC, waist circumference; FG, fasting glucose; SBP, systolic blood pressure; DBP, diastolic blood pressure, HDL-C, HDL cholesterol; LDL-C, LDL cholesterol; hs-CRP, high-sensitivity C-reactive protein; IAF, intraabdominal fat; SAF, subcutaneous abdominal fat.

${ }^{\star} P<0.05$.

Table 3 Prediction of MetS components and adiposity measurements to In(HOMA-IR) among apparently healthy obese women (n 96, mean age 42 years), Busan, South Korea

\begin{tabular}{llrrr}
\hline & Predictor (unit) & Beta & \multicolumn{1}{c}{ SE } & \multicolumn{1}{c}{$P$} \\
\hline Model 1 & FG $(1 \mathrm{mmol} / \mathrm{l})$ & 0.41 & 0.084 & $<0.001$ \\
& TAG $(1 \mathrm{mmol} / \mathrm{l})$ & 0.31 & 0.082 & $<0.001$ \\
& WC $(1 \mathrm{~cm})$ & 0.02 & 0.008 & 0.006 \\
& Age $(1 \mathrm{year})$ & -0.01 & 0.005 & 0.011 \\
Model 2 & FG (1 mmol/l) & 0.44 & 0.085 & $<0.001$ \\
& TAG (1 mmol/l) & 0.38 & 0.082 & $<0.001$ \\
Model 3 & Age (1 year) & -0.02 & 0.006 & 0.007 \\
& FG (1 mmol/l) & 0.41 & 0.085 & $<0.001$ \\
& TAG (1 mmol/l) & 0.33 & 0.083 & $<0.001$ \\
& Age (1 year) & -0.01 & 0.005 & 0.012 \\
& Android fat (1\%) & 0.03 & 0.011 & 0.023
\end{tabular}

MetS, metabolic syndrome; HOMA-IR, homeostasis model assessment of insulin resistance; WC, waist circumference.

$R^{2}=0.42$ for model $1 ; R^{2}=0.40$ for model $2 ; R^{2}=0.43$ for model 3 .

Excluded variables were systolic blood pressure (SBP), diastolic blood pressure (DBP) and HDL cholesterol (HDL-C) in model 1; SBP, DBP, HDL$\mathrm{C}$, intra-abdominal fat (IAF) and subcutaneous abdominal fat (SAF) areas in model 2; and SBP, DBP and HDL-C in model 3.

despite weight loss, while insulin sensitivity for women with IR improves after weight reduction ${ }^{(6)}$. Therefore, different treatment options may be required for obese women with IR and for those without IR.

In the present study, in ninety-six apparently healthy obese Korean women, high FG and high TAG better predicted IR compared with high BP and low HDL-C. According to the multiple regression analyses, FG and TAG were consistently associated with $\ln$ (HOMA-IR) regardless of abdominal obesity, while BP and HDL-C were not.

In the context of diagnostic utility of MetS criteria, $28 \%$ of obese women with high FG, 33\% of obese women with high TAG, and less than $20 \%$ of obese women with low HDL-C or high BP were insulin-resistant, whereas over $90 \%$ of those without these criteria were not insulinresistant. The positive likelihood ratio indicates that if the probability of having IR prior to doing serum FG or TAG tests is $50 \%$ for an obese woman, the probability of having IR increases to $80 \%$ or $75 \%$ when she is found to have high FG or high TAG values, respectively, after those tests are done. The negative likelihood ratio indicates that the probability of absence of IR decreases to $33 \%$ or $38 \%$, respectively, when she is found to have normal values of the two tests. In contrast, the post-test probability increases only to $63 \%$ if low HDL-C or high BP is applied. Altogether, these results support that, among MetS criteria, high FG and high TAG seem to be more useful indicators to identify individuals with IR among obese women. In contrast, the clinical usefulness of high BP and low HDL-C criteria as predictors of IR seems to be low.

Studies have provided complicated pathophysiological mechanisms to explain the relationship between IR and increased TAG level. IR results in reduced activity of endothelial-bound lipoprotein lipase (LPL), which contributes to impaired TAG hydrolysis and uptake of chylomicron and VLDL lipids by muscle and adipose tissue. Insulin-resistant states also result in increased levels of apoCIII, an inhibitor of LPL, and impaired apoE-mediated receptor uptake of TAG-rich lipoproteins and their lipolytic remnants ${ }^{(12)}$.

The ability of WC to predict IR, as measured by HOMAIR, in current subjects was not inferior to that of other abdominal adiposity measurements assessed using more sophisticated tools. Several studies have also demonstrated that no one particular measurement of abdominal adiposity is superior to others in its association with $\mathrm{IR}^{(13)}$. 
Table 4 Odds ratios of each MetS criterion for IR among apparently healthy obese women ( $n$ 96, mean age 42 years), Busan, South Korea

\begin{tabular}{|c|c|c|c|c|c|c|}
\hline & \multirow{2}{*}{$\begin{array}{l}\text { Women without } \\
\text { IR }(n \text { 85) }(\%)\end{array}$} & \multirow{2}{*}{$\begin{array}{l}\text { Women with } \\
\text { IR }(n 11)(\%)\end{array}$} & \multicolumn{2}{|c|}{ Crude } & \multicolumn{2}{|c|}{ Adjusted } \\
\hline & & & OR & $95 \% \mathrm{Cl}$ & OR & $95 \% \mathrm{Cl}$ \\
\hline $\mathrm{FG} \geq 5.6 v .<5.6 \mathrm{mmol} / \mathrm{l}$ & $14 \cdot 1$ & $54 \cdot 5$ & $7 \cdot 3$ & $1 \cdot 9,27 \cdot 7$ & $8 \cdot 6+$ & $1 \cdot 8,41 \cdot 8$ \\
\hline TAG $\geq 1.69 v .<1.69 \mathrm{mmol} / \mathrm{l}$ & $15 \cdot 3$ & $45 \cdot 5$ & $4 \cdot 6$ & $1 \cdot 2,17 \cdot 3$ & $2 \cdot 3 t$ & $0.5,11 \cdot 6$ \\
\hline WC ( $1 \mathrm{~cm}$ increase) & - & - & $1 \cdot 1$ & $1 \cdot 0,1 \cdot 2$ & $1 \cdot 1 \dagger$ & $1 \cdot 0,1 \cdot 2$ \\
\hline $\mathrm{HDL}-\mathrm{C}<1.29 \mathrm{v} . \geq 1.29 \mathrm{mmol} / \mathrm{l}$ & $36 \cdot 5$ & $63 \cdot 6$ & $3 \cdot 0$ & $0 \cdot 8,11 \cdot 2$ & $3.0 t$ & $0 \cdot 6,16 \cdot 0$ \\
\hline $\mathrm{BP} \geq 130 / 85$ v. $<130 / 85 \mathrm{mmHg}$ & $35 \cdot 7$ & $63 \cdot 6$ & $3 \cdot 2$ & $0.9,11 \cdot 6$ & $2 \cdot 0+$ & $0 \cdot 4,9 \cdot 3$ \\
\hline Fulfilled MetS & $26 \cdot 2$ & $81 \cdot 8$ & $12 \cdot 7$ & $2 \cdot 5,63 \cdot 3$ & $13 \cdot 5 \ddagger$ & $2 \cdot 6,69 \cdot 1$ \\
\hline
\end{tabular}

MetS, metabolic syndrome; IR, insulin resistance; FG, fasting glucose; WC, waist circumference; HDL-C, HDL cholesterol; BP, blood pressure.

IR was defined as HOMA-IR (homeostasis model assessment of IR) $\geq 3.04$.

MetS was defined when two out of the four criteria of the MetS were fulfilled.

tLogistic regression model in which age and five metabolic syndrome criteria were included as predictors.

łLogistic regression model in which age and the presence/absence of MetS were included as predictors.

Table 5 Kappa values, sensitivity, specificity, likelihood ratios and predictive values of MetS criteria for detecting IR in apparently healthy obese women ( $n 96$, mean age 42 years), Busan, South Korea

\begin{tabular}{|c|c|c|c|c|c|c|c|c|c|c|c|}
\hline \multirow[b]{2}{*}{ Criterion } & \multirow[b]{2}{*}{$\kappa$} & \multicolumn{2}{|c|}{ Sensitivity } & \multicolumn{2}{|c|}{ Specificity } & \multicolumn{2}{|c|}{$+\mathrm{LR}$} & \multicolumn{2}{|c|}{$-\mathrm{LR}$} & \multirow[b]{2}{*}{$+\mathrm{PV}(\%)$} & \multirow[b]{2}{*}{-PV (\%) } \\
\hline & & $\%$ & $95 \% \mathrm{Cl}$ & $\%$ & $95 \% \mathrm{Cl}$ & $\%$ & $95 \% \mathrm{Cl}$ & $\%$ & $95 \% \mathrm{Cl}$ & & \\
\hline $\mathrm{FG} \geq 5.6 \mathrm{mmol} / \mathrm{l}$ & $0 \cdot 3^{*}$ & 55 & 24,83 & 86 & 77,93 & 3.9 & $2 \cdot 2,6 \cdot 7$ & 0.5 & $0 \cdot 2,1 \cdot 2$ & 33 & 94 \\
\hline $\mathrm{TAG} \geq 1.69 \mathrm{mmol} / \mathrm{l}$ & $0 \cdot 2^{*}$ & 45 & 17,77 & 85 & 75,92 & $3 \cdot 0$ & $1 \cdot 5,5 \cdot 7$ & 0.6 & $0 \cdot 3,1 \cdot 3$ & 28 & 92 \\
\hline $\mathrm{BP} \geq 130 / 85 \mathrm{mmHg}$ & $0 \cdot 1$ & 64 & 31,89 & 64 & 53,74 & $1 \cdot 8$ & $1 \cdot 1,2 \cdot 9$ & 0.6 & $0 \cdot 2,1 \cdot 3$ & 19 & 93 \\
\hline $\mathrm{HDL}-\mathrm{C}<1.29 \mathrm{mmol} / \mathrm{l}$ & $0 \cdot 1$ & 64 & 31,89 & 64 & 52,74 & $1 \cdot 7$ & $1 \cdot 1,2 \cdot 8$ & 0.6 & $0 \cdot 2,1 \cdot 3$ & 18 & 93 \\
\hline Fulfilled MetS criteria & $0 \cdot 3^{*}$ & 82 & 48,97 & 74 & 63,83 & $3 \cdot 1$ & $2 \cdot 3,4 \cdot 2$ & 0.3 & $0.1,0.9$ & 29 & 97 \\
\hline
\end{tabular}

MetS, metabolic syndrome; IR, insulin resistance; LR, likelihood ratio; PV, predictive value; FG, fasting glucose; BP, blood pressure; HDL-C, HDL cholesterol. MetS was defined when two out of the four criteria were fulfilled.

IR was defined as HOMA-IR (homeostasis model assessment of IR) $\geq 3.04$. ${ }^{\star} P<0.05$.

In the current obese Korean women, those in the metabolically healthy group, i.e. those without IR ( $89 \%$ of current subjects), were more likely to have favourable values of metabolic components and lower visceral fat content than the metabolically unhealthy group, while hs-CRP was not significantly different between the two groups. In contrast, previous studies have shown significant difference in hs-CRP level between the two groups $^{(14)}$. Probably the discrepancy in the results may relate to differences in ethnicities, measures of assessing IR, or definitions of metabolic health status.

The relationship between $\ln$ (HOMA-IR) and $\ln$ (TAG: HDL), another surrogate marker of IR, reveals a very similar correlation to that found among postmenopausal obese Caucasian women in a previous study ${ }^{(15)}$. However, the correlation level seems not to be higher compared with the correlation with FG or TAG.

Several limitations of the present study should be considered. As the participants were obese Korean women and were not randomly selected, and the study sample size was also small, generalization to men, other ethnicities, individuals with normal weight or even obese women with different characteristics may not be made. In addition, although HOMA-IR is frequently used to evaluate IR in population-based studies because of its simplicity and acceptable validity ${ }^{(16)}$, IR was not assessed using the more valid clamp method. Therefore, the surrogate marker of IR using HOMA-IR could possibly lead to the misclassification of women as having IR or not. Finally, the causality is uncertain because the study was cross-sectional in nature.

In conclusion, among the IDF-defined MetS criteria, high FG and high TAG seem to be most suitable for identifying obese women with IR. Further validations regarding the relationship between the MetS criteria and IR are required.

\section{Acknowledgements}

The work was supported by the NS Medicom Company, South Korea. There is no conflict of interest to declare.

\section{References}

1. Reaven G (2004) The metabolic syndrome or the insulin resistance syndrome? Different names, different concepts, and different goals. Endocrinol Metab Clin North Am 33, 283-303.

2. Expert Panel on Detection, Evaluation, and Treatment of High Blood Cholesterol in Adults (2001) Executive Summary of The Third Report of The National Cholesterol Education Program (NCEP) Expert Panel on Detection, Evaluation, And Treatment of High Blood Cholesterol In Adults (Adult Treatment Panel III). JAMA 285, 2486-2497.

3. Alberti KG, Zimmet P \& Shaw J (2006) Metabolic syndrome a new world-wide definition. A Consensus Statement from the International Diabetes Federation. Diabet Med 23, 469-480.

4. Despres JP (2001) Health consequences of visceral obesity. Ann Med 33, 534-541. 
5. Perseghin G (2008) Is a nutritional therapeutic approach unsuitable for metabolically healthy but obese women? Diabetologia 51, 1567-1569.

6. Karelis AD, Messier V, Brochu M \& Rabasa-Lhoret R (2008) Metabolically healthy but obese women: effect of an energy-restricted diet. Diabetologia 51, 1752-1754.

7. McLaughlin T, Abbasi F, Cheal K, Chu J, Lamendola C \& Reaven G (2003) Use of metabolic markers to identify overweight individuals who are insulin resistant. Ann Intern Med 139, 802-809.

8. Hsieh CH, Pei D, Hung YJ, Kuo SW, He CT, Lee CH \& Wu CZ (2008) Identifying subjects with insulin resistance by using the modified criteria of metabolic syndrome. J Korean Med Sci 23, 465-469.

9. Matthews DR, Hosker JP, Rudenski AS, Naylor BA, Treacher DF \& Turner RC (1985) Homeostasis model assessment: insulin resistance and $\beta$-cell function from fasting plasma glucose and insulin concentrations in man. Diabetologia 28, 412-419.

10. Lee S, Choi S, Kim HJ, Chung YS, Lee KW, Lee HC, Huh KB \& Kim DJ (2006) Cutoff values of surrogate measures of insulin resistance for metabolic syndrome in Korean nondiabetic adults. J Korean Med Sci 21, 695-700.
11. Bloomgarden ZT (2004) Definitions of the insulin resistance syndrome: the 1st World Congress on the Insulin Resistance Syndrome. Diabetes Care 27, 824-830.

12. Krauss RM \& Siri PW (2004) Metabolic abnormalities: triglyceride and low-density lipoprotein. Endocrinol Metab Clin North Am 33, 405-415.

13. Lee K, Lee S \& Kim YJ (2008) Waist circumference, dualenergy X-ray absorptiometrically measured abdominal adiposity, and computed tomographically derived intraabdominal fat area on detecting metabolic risk factors in obese women. Nutrition 24, 625-631.

14. Karelis AD (2008) Metabolically healthy but obese individuals. Lancet 372, 1281-1283.

15. Karelis AD, Pasternyk SM, Messier L, St-Pierre DH, Lavoie JM, Garrel D \& Rabasa-Lhoret R (2007) Relationship between insulin sensitivity and the triglycerideHDL-C ratio in overweight and obese postmenopausal women: a MONET study. Appl Physiol Nutr Metab 32, 1089-1096.

16. Muniyappa R, Lee S, Chen H \& Quon MJ (2008) Current approaches for assessing insulin sensitivity and resistance in vivo: advantages, limitations, and appropriate usage. $\mathrm{Am}$ J Physiol Endocrinol Metab 294, E15-E26. 